\title{
Meeting Highlights of the I I th Annual Scientific Sessions of the Society for Cardiovascular Magnetic Resonance, Los Angeles, February I-3, 2008
}

\author{
Matthias G Friedrich*1, Warren J Manning2, Thoralf Niendorf3, \\ Charles B Higgins ${ }^{4}$, Albert de Roos $^{5}$ and Stefan Neubauer ${ }^{6}$
}

\begin{abstract}
Address: ${ }^{1}$ Stephenson CMR Centre at the Libin Cardiovascular Institute of Alberta, University of Calgary, Calgary, AB, Canada, T2N 2T9, ${ }^{2} \mathrm{Beth}$ Israel Deaconess Medical Center, Boston, MA, USA, ${ }^{3}$ Department of Diagnostic Radiology, Technical University, Aachen, Germany, ${ }^{4}$ Department of Radiology, University of California School of Medicine, San Francisco, San Francisco, CA, USA, ${ }^{5}$ Leiden University Medical Center, Leiden, the Netherlands and ${ }^{6}$ Oxford University, Oxford, UK

* Corresponding author
\end{abstract}

from I th Annual SCMR Scientific Sessions

Los Angeles, CA, USA. I-3 February 2008

Published: 22 October 2008

Journal of Cardiovascular Magnetic Resonance 2008, I0(SuppI I):II doi:I0.I I86/I532-429X-I0-SI-II

This article is available from: http://jcmr-online.com/content/I0/SI/II

(c) 2008 Friedrich et al; licensee BioMed Central Ltd.

\begin{abstract}
This paper features the most interesting presentations and discussions of the 2008 Annual Sessions of the Society for Cardiovascular Magnetic Resonance, which were held in Los Angeles from February I to 3, 2008.

With more than 1100 attendees, this was the largest of the SCMR meetings ever. Among this year's highlights were scientific reports on CMR-based risk assessment, non-contrast tissue characterization, $3 \mathrm{~T}$ data, and interventional CMR.
\end{abstract}

\section{Introduction}

This year's Annual Sessions of the Society for Cardiovascular Magnetic Resonance (SCMR) were held from February 1 to 3 in Los Angeles, California.

The meeting attracted a record number of more than 1100 attendees. The main program was the largest on CMR ever and featured a total of 41 sessions, among them 15 for oral abstract sessions, nine clinical, five on experimental $M R$, four with case discussions, two offering an informal "Coffee with the Expert", two on interventional sessions, two on congenital CMR, one on political aspects, and one for trainees. 154 invited talks provided state-of-the-art review on experimental and clinical CMR protocols, controversial aspects and new data. Among this year's areas of interest were risk stratification of CMR, interventional $\mathrm{CMR}$, molecular CMR, and tissue characterization in nonischemic cardiomyopathies. Among 532 submitted abstracts, 291 were accepted as posters, 12 as moderated posters, and 102 for oral presentations.

\section{Workshops}

Preceding the main meeting, an introductory physicians' CMR course (Chairs: Drs. David Bluemke and Peter Buser, University Hospital, Basel), a workshop on CMR at $3 \mathrm{~T}$ (Chair: Dr. Gerald Pohost), and a workshop on basic and experimental research in CMR (Chairs: Drs. Sodickson and Niendorf) were held. 
A 3-day technologists' meeting (Chair Mary Watkins, Washington University, St. Louis, Missouri) was offered parallel to the main meeting.

\section{Clinical CMR}

Cardiovascular risk assessment: defining better therapeutic targets by $C M R$

Dr. Peter Libby from Harvard Medical School in Boston opened this year's plenary Focus Session and set the stage for talks on the spectrum of CMR-derived markers of atherosclerotic risk, ranging from plaque structure to metabolic changes by Dr. Zahi Fayad (Mount Sinai School of Medicine, New York) and Dr. Hildo Lamb (Leiden University Medical Center, Leiden, NL). Dr. Robert Kloner (Keck School of Medicine, Los Angeles, CA) introduced the audience to the targets for secondary prevention, which were addressed by Dr. Joao Lima (Johns Hopkins University, Baltimore, MD) and the concluding talk from Dr. Dudley Pennell (Royal Brompton Hospital, London, UK), who emphasized prognostic data of CMR based on in vivo tissue pathology.

\section{Young Investigator Award Session - clinical}

The opening plenary session was followed by the Young Investigator Oral Clinical Presentations. These represented the abstracts receiving the highest overall grading among individuals within 5 years of completion of their training. Opening this very exciting session was the award winner, Dr. Joseph Selvanayagam (100 [A1]) from Oxford who presented data on the prognostic importance of late gadolinium enhancement (LGE). In a study of 152 patients undergoing LGE prior to their first percutaneous coronary intervention or coronary artery bypass surgery, they found the presence of new LGE to be the single independent predictor of major endpoints (death, non-fatal myocardial infarction, ventricular arrhythmia terminated by ICD, unstable angina, and heart failure. Dr. Qi Yang (102 [A3]) from Beijing presented a study on the use of contrast enhanced inversion recovery whole heart coronary MRI at $3 \mathrm{~T}$ in 22 patients. Compared with x-ray angiography, MRI had an accuracy exceeding 95\%. Also using 3 T, Dr. Allison Hays (103 [A4]) of Johns Hopkins University then presented coronary artery velocity data from a study of rest and handgrip stress in 9 healthy subjects. The mean increase in peak velocity was nearly $40 \%$.

\section{CMR of myocardial viability}

As for the past years, CMR assessment of viability was a major focus of the SCMR annual meeting. The session began with a technical report by Dr. Amol Pedneker (112 [A13]) demonstrating that a mid-wall LGE pattern can be artifactually created when using a low-high phase encoding order and the use of linear phase encoding order is preferred. Dr. Jose Rodriguez (113 [A14]) from Northwestern presented data on microvascular obstruction using contrast-enhanced CMR as areas of hypoperfusion in the infarct zone. Among patients followed during their first week, at 3 months, and 1 year following infarction, there was progressive reduction of the hypoenhancement volume. In addition the volume of hypoenhancement progressively declined when assessed from 1 minute to 25 minutes after contrast injection. The best predictors of end-systolic volume and ejection fraction at 1 year were LGE at 15 minutes and the volume of hypoenhancement at 10 and 15 minutes. Sharon Kirschbaum (116 [A17]) then presented comparative LGE and both 5 and $10 \mathrm{mcg} /$ $\mathrm{kg} /$ min dobutamine viability data regarding chronic total coronary occlusion. Dysfunctional segments with $<25 \%$, $26-50 \%$ and $>75 \%$ transmural LGE demonstrated similar improvement in systolic wall thickening with 5 and 10 $\mathrm{mcg} / \mathrm{kg} / \mathrm{min}$ of dobutamine while $50-75 \%$ transmural LGE had improved wall thickening with $10 \mathrm{mcg} / \mathrm{kg} / \mathrm{min}$. The session concluded with a presentation by Dr. GrosseWortmann (117 [A18]) examining the inversion time differences of the left and right ventricle, concluding that the difference was likely related to partial volume effect from the this right ventricle.

\section{CMR of non-ischemic heart disease}

Two sessions focused on topics related to experimental and clinical CMR approaches to Non-Ischemic Heart Disease.

In a study of 116 patients with suspected LV thrombus, Dr. Michael Ross and his team from Cornell University and Duke University Center provided strong evidence that CMR detects intracavitary thrombi more sensitively than contrast echo (202 [A63]). Among several presentations on tissue characterization, Peter Hunold from the University Hospital, Essen, Germany showed data on 5676 patients assessed with CMR. Of those, 1905 patients had cardiac scars as defined by areas with late enhancement (205 [A66]). Visualization of scars not only was useful to differentiate ischemic from non-ischemic injury, but also to identify the cause of non-ischemic injury, such as HCM, myocarditis, DCM and storage diseases.

Dr. Anja Zagrosek of Franz-Volhard-Klinik, Berlin reported on CMR imaging of myocardial edema in 21 patients with acute myocarditis. The authors not only observed edema as reflected by an increase of normalized global myocardial signal intensity in T2-weighted images, but also its decline, which was accompanied by a decrease of LV mass during the follow-up of 9 months (224 [A85]). Finally, Tjeerd Germans and co-workers from centers in Amsterdam, Utrecht and Maastricht (all in The Netherlands) could show that CMR-derived strain and strain rate detects systolic and diastolic dysfunction in $28 \mathrm{HCM}$ mutation carriers without LV hypertrophy (227 [A88]). 


\section{CMR data on prognosis}

With the expansion of CMR centers, there has been considerable interest in the prognostic value of CMR. Dr. Dall'Armellina (120 [A21]) from Wake Forest University presented 5 year prognostic data on 200 consecutive patients with LVEF $<55 \%$ undergoing dobutamine stress CMR. Among those with an LVEF of $40-55 \%$, dobutamine induced changes in LV wall motion score predicted adverse cardiac events beyond resting LVEF. Dr. Pilz (121 [A22]) from the University of Munich then presented one year prognosis data on 218 patients undergoing adenosine stress CMR. Patients with a normal adenosine stress CMR had an excellent prognosis (no events in 99\%). Dr. Nijveldt (123 [A24]) from the University Medical Center, Amsterdam then presented data from 60 patients with the first acute myocardial infarction. LGE microvascular obstruction was associated with wall thinning and reduced improvement in wall thickening, independent of LGE transmurality. The results of the first multicenter international LGE study were then presented by Dr. Lenge (124 [A25]) from Baylor College of Medicine. Of 183 patients referred for revascularization, the transmural extent of LGE was inversely related to functional recovery with $72 \%$ of dysfunctional segments without LGE demonstrating recovery vs. only $17 \%$ of segments with near complete transmural LGE.

\section{Progress in coronary CMR imaging}

While coronary multidetector computed tomography (MDCT) angiography has captured the attention of many, increased attention has been turning to coronary MRI due to the lack of ionizing radiation and iodinated contrast. The coronary MRI session started with a presentation by Dr. Liu (126 [A27]) (Northwestern University, Chicago, IL) who performed coronary MDCT angiography and whole heart coronary MRI in 18 patients with increased epicardial calcium. Using oral beta blockers and nitroglycerin in both groups, whole heart coronary MRI was found to be superior to 64-slice MDCT. In the same session, Dr. Bernhard Gerber (128 [A29]) (Cliniques Universitaires, Brussels, BE) presented data on a head-to-head comparison of whole-heart coronary MRI and 40-64 slice MDCT. While MDCT had greater success in acquiring interpretable images, among interpretable segments and vessels, coronary MRI and MDCT had similar accuracy. Finally, Dr. Macdedo (127 [A28]) from Johns Hopkins presented provocative data from the Multi-Ethnic Study of Atherosclerosis (MESA) that coronary wall thickness was increased among their subjects with $\geq 2$ CAD risk factors. These same investigators also presented data that lower heart rate and longer rest periods were associated with improved coronary wall imaging.

\section{Toward a better understanding of cardiovascular risk}

The cardiovascular risk session started with a presentation by Dr. Michael Chuang (133 [A34]) (Beth Israel Deaconess Medical Center, Boston, MD) on lean and obese subjects from the Framingham Heart Study Offspring cohort. Across all subjects, myocardial contraction fraction (ratio of LV stroke volume to myocardial volume) was greater in women and LV ejection fraction was greater in obese vs. lean subjects. There were two CMR spectroscopy (CMRS) abstracts: Dr. Rutger van der Meer (134 [A35], 135 [A36]) (Leiden University Medical Center, Leiden, NL) then presented provocative CMRS data in 43 healthy male subjects showing that age was significantly correlated with interventricular septal triglyceride content. These same investigators also demonstrated data that a short-term excessive dietary fat intake led to increased hepatic triglyceride content, but no change in myocardial triglyceride content. The session concluded with a presentation by Dr. Oliver Rider (137 [A38]) (University of Oxford, UK) who provided CMR evidence that increased body mass index correlated with positively with $\mathrm{LV}$ and RV mass. With the transition from normal to overweight, and an increase in serum leptin, there was an increase in LV and RV mass, but no change in LV and RV volumes.

\section{CMR endpoints and clinical trials}

The excellent interstudy reproducibility and lack of ionizing radiation with $\mathrm{CMR}$ renders it the most attractive imaging technique for clinical trials. As Dr. Steven Dymarkowski from University Hospital, Leuven, BE pointed out, the current steady state free precession (SSFP) cine MR provides even better interstudy reproducibility than has been previously reported for gradient echo cine CMR. Dr. Nathaniel Reichek of St. Francis Hospital, New York indicated that the better interobserver and interstudy reproducibility of CMR for quantifying LV mass, compared to echocardiography, permits a considerable reduction in sample size of approximately 50 patients vs. 1000 patients to indicate a drug effect. Dr. Raymond Kim reported results of a multicenter study on LGE in acute myocardial infarction. LGE CMR had a 94\% accuracy for showing infarct in proper distribution of the culprit artery as verified by angiography.

\section{Moderated poster presentations - clinical}

As always, the moderated poster sessions were a highlight of the meeting, providing the opportunity for the authors to present their work to attendees in an intimate setting accompanied by wine and cheese. Dr. Meear Sekar (301 [A104]) of Northwestern University, Chicago presented quantitative data comparing Doppler with CMR phase velocity assessment of aortic regurgitation. Dr. Noriko Oyama (302 [A105]) from the Beth Israel Deaconess Medical Center, Boston reported on the novel finding of relationship of CMR epicardial fat to gender and age. This 
was followed by the award-winning presentation of Dr. Mirja Neizel (303 [A106]) from University Hospital, Heidelberg, Germany on strain-encoded (SENC) imaging of myocardial function after acute myocardial infarction. Among 14 patients with acute infarction, quantitative circumferential strain was significantly impaired in visually hypo- and akinetic regions on cine CMR (vs. normokinetic segments). Dr. He (304 [A107]) of the Royal Brompton Hospital, London, UK presented data from their extensive experience with thalassemia patients and T2* assessment of myocardial iron. Curve fitting using a truncation model was the most accurate and most reproducible. The session finished with a presentation by Dr. Flett (305 [A108]) from the Heart Hospital of London on the use of real time cine CMR to identify patients with marked septal flattening as being a very specific sign for patients with pericardial constriction.

\section{CMR vs. CT in vascular and coronary disease}

The value of coronary MDCT was discussed by Dr. Matthew Budoff (David Geffen School of Medicine, Los Angeles, CA). MDCT appears to be an excellent technique for excluding coronary artery stenosis in selected patients. The assessment of plaque characteristics in coronary vessels by CT however is still limited and there is a lack of clinical validation data. MRI is a versatile tool to assess many features of atherosclerosis. As an example, MRI techniques are being developed for the in vivo assessment of plaque vulnerability by investigating mechanical stress on the fibrous cap. Both CT and MRI are still hampered for a thorough assessment of plaque components and vulnerability in coronary vessels by insufficient spatial resolution and limitations due to motion artifacts. Vascular wall imaging in larger vessels (carotids, aorta) is promising for serial monitoring of atherosclerosis and the evaluation of therapy.

\section{First-pass perfusion $C M R$}

In the session on the current status of perfusion-CMR, Dr. Norbert Wilke (University of Florida, Gainsville, FL) demonstrated the performance and current limitations of $3 \mathrm{~T}$ perfusion imaging versus 1.5 T. Dr. Peter Gatehouse (Royal Brompton Hospital, London, UK) addressed the potential as well as the so far unresolved issues of absolute quantification of myocardial perfusion calculated from first-pass data.

More clinical talks focused on the diagnostic and prognostic performance power of established CMR perfusion protocols. Dr. Jürg Schwitter (Universitätsspital Zurich, Switzerland) highlighted the international multicenter, multivendor trial called MR-IMPACT (Magnetic Resonance Imaging for Myocardial Perfusion Assessment in Coronary artery disease Trial). This largest trial performed so far in 18 centers in Europe and the US was recently pub- lished [1] and demonstrated an excellent and superior diagnostic performance of CMR perfusion vs. single-photon emission computed tomography utilizing invasive coronary angiography as the standard of reference. Dr. Eike Nagel (King's College, London, UK) presented an overview of the growing body of literature on the prognostic value of first-pass CMR perfusion. In fact, a normal of first-pass CMR perfusion or dobutamine stress CMR test goes along with an excellent prognosis matching that of healthy subjects.

Finally, Dr. Rohan Dharmakumar (Northwestern University, Chicago, IL) reviewed experimental and preliminary human experiments exploring an oxygen-level dependent CMR technique to detect myocardial ischemia.

\section{Vascular MR}

Continuing developments in the field of gradient hardware and pulse sequence design, parallel imaging, the migration to higher field strengths and the clinical introduction of novel intra-vascular contrast agents have advanced the field of vascular MR significantly over the past years. The combination of these advances allow for acquiring time-resolved, high spatial resolution MR angiograms with small doses of contrast agents. The most significant advance, however, has been the emerging ability of contrast-enhanced MRA to provide information on organ perfusion, in addition to vascular morphology. Quantitative perfusion data can be obtained by tracking contrast enhancement in main organ arteries in relation to the enhancement of the target organ itself. An important potential application is the comprehensive assessment of renovascular disease: While MRA can detect or rule out renal artery stenosis, organ enhancement techniques enable the assessment of renal parenchymal perfusion, all with just a small bolus of contrast agent.

\section{Experimental and basic/non-clinical CMR}

CMR@3.0 T - solved and unsolved problems

The capabilities of MR technology at high-field promise to translate into extra diagnostic value in CMR imaging, not only by streamlining structural and functional imaging, but also by facilitating targeted tissue characterization and improving access to physiological information. It stands to reason that all these developments might culminate in a paradigm shift for the translation of basic research into clinical MRI, although high-field MRI has been regarded as one of the most challenging MRI applications, because of constraints associated with magnetic field inhomogeneities and localized tissue heating due to RF power deposition, as emphasized by Dr. Michael Jerosch-Herold (Brigham \& Women's Hospital, Harvard Medical School, Boston, MA, USA). 
High-field imaging strategies are also appealing for the capture of dynamic events through the powerful synergy with parallel imaging strategies. The merits of otherwise unattainable SNR combined with improvements in acquisition speed promise to further advance real time imaging, as discussed by Dr. Krishna Nayak (University of Southern California, Los Angeles, CA). Despite some appreciable tradeoffs associated with high-field imaging - e.g. the quality degradation of steady-state free precession sequences - the promise of high-field real time CMR has been recognized both as a driver and as a beneficiary of the move towards $3.0 \mathrm{~T}$ many-channel MR systems. The benefits of high-field MR may be exploited in numerous ways as demonstrated for MR angiography by Paul Finn (University of California, Los Angeles, CA); to facilitate continuous volumetric acquisitions with previously unattainable temporal resolution; or to increase anatomic coverage or spatial resolution within a given imaging time, allowing comprehensive scanning of any target vessel territory, while avoiding the frequently-encountered difficulty of truncated anatomy; or to improve temporal resolution of time-resolved MRA down to 2-4 s to clearly distinguish arterial from venous phases, without substantially sacrificing the spatial resolution; or to evaluate contrast dynamics. Initial experience at 3.0 T suggests that higher field strengths in conjunction with tailored RF coil array designs may enable coronary MRA with sub-millimeter spatial resolution together with otherwise unattainable volume coverage as reported by Dr. Debiao Li (Northwestern University, Chicago, IL, USA). However, the issue of the off-resonance sensitivity of SSFP-techniques remains a concern, which has prompted the comeback of gradient-echo imaging techniques in coronary artery imaging.

\section{Parallel and rapid imaging}

Imaging speed becomes an increasingly significant limitation as new CMR applications are explored. The advent of parallel imaging introduced a new means of acceleration. Dr. Jeffrey Tsao (Novartis Inc, Cambridge, MA, USA) pointed out that the resulting efficiency and speed gain can be clinically exploited in a number of ways, ranging from enhancement of spatial and temporal resolution, efficient volumetric imaging, to streamlining of CVMR workflow. He demonstrated that, for dynamic imaging applications including left ventricular function or local wall thickness assessment, first pass perfusion imaging, contrast enhanced MRA and flow imaging, imaging techniques that exploit spatio-temporal correlations in the data may be a preferred means of achieving high accelerations, given their favourable SNR behaviour as compared to sensitivity encoding based approaches. Of course, these "spatio-temporal" acceleration techniques may be combined with parallel imaging for further improvements in imaging speed and/or coverage.
Dr. Sebastian Kozerke (University and ETH Zurich, Zurich, Switzerland) reported that high levels of acceleration have recently become possible with many-receiver MR-systems. He described the critical role of cardiac surface RF coil arrays, a key enabling factor for high accelerations; together with prevailing trends towards $32+$ receiver channels and high-field imaging (i.e. $3 \mathrm{~T}$ and beyond). The incremental diagnostic value promised by highly accelerated parallel imaging and ergonomic requirements are likely to motivate technological developments, including innovative cabling methods, or even wireless coil connectivity. A move towards 64-bit reconstruction hardware and parallel computation has been recognized as an important step in overcoming current memory and performance constraints on image reconstruction for manychannel volumetric acquisitions. An important development in this context is the move towards coil array compression to reduce the computational load and reconstruction times.

Dr. Vivek Muthurangu (University College, London, UK) provided several examples of highly parallel imaging strategies, including single breath-hold whole heart cine MRI for rapid assessment of cardiac function, which improves patient comfort and eliminates the risk of slice registration errors.

Dr. Peter Kellman (National Heart, Lung and Blood Institute, Bethesda, MD, USA) discussed clinical cardiac applications including myocardial perfusion and delayed enhancement imaging. He illustrated how parallel imaging could provide improved coverage and facilitate the suppression of "dark rim" artifacts in myocardial perfusion imaging, which may mimic subendocardial perfusion deficits. He also presented an automatic freebreathing, late enhancement imaging method with improved SNR, rendering it particularly suitable for the assessment of patients with arrhythmia or limited compliance.

Dr. Gerhard Laub (Siemens, Medical Systems, Los Angeles, CA, USA) presented time-resolved contrast enhanced MRA as another imaging application that benefits from accelerated imaging. He discussed how parallel imaging can be used to achieve improved vessel sharpness and delineation, and improved separation of arterial and venous phases.

\section{New RF coil design}

In CMR, where maximum spatial and temporal resolution is needed, the design of optimized RF-coils is particularly important. Designs presented in this session included coil arrays for proton and non-proton CMR, with a trend to increasing number of array elements. Improvements in imaging speed or spatial resolution were demonstrated. In 
addition to phased array receiver technology, parallel RFtransmission may in particular represent a major development to overcome SAR-limitations at ultra-high magnetic fields, and to reduce imaging time by improved volume selection.

\section{Metabolic CMR studies}

CMRS is the only technique that allows for a non-invasive assessment of metabolism in the heart. While a number of proton and phosphorous-31 CMRS studies have been reported in humans and animal models showing feasibility and providing early insights as proof of principle, a novel approach to increase the sensitivity of Carbon-13 CMRS by five orders of magnitude, using hyperpolarizing Carbon-13 compounds, was demonstrated in two presentations (Arnaud Comment, Ecole Polytechnique Fédérale de Lausanne, Lausanne, Switzerland and Damian Tyler, University of Oxford, UK). Although the application of this technique in animal models showed its impressive potential, the transfer to clinical use remains yet to be demonstrated.

\section{Young Investigator Award Session (non-clinical)}

Interesting papers presented at this session included reports on advancements in small-field-of-view black blood imaging using fast spin echo sequences, acceleration of 3-dimensional tagging, and diffusion MRI to assess fiber changes occurring during development from fetal to adult heart. Of particular relevance was a study presented by Sebastiaan Hammer from Leiden University Medical Center, Leiden, The Netherlands (106 [A7]): Combining proton CMRS and CMR imaging, the researchers could demonstrate that substantial weight loss in obese patients with Type 2 diabetes mellitus not only eliminated the need for additional insulin supplementation, but also resulted in improved left ventricular function and reduced myocardial triglyceride levels. This work demonstrates the potential of CMR to combine metabolic and functional information for a more comprehensive diagnosis of heart disease.

\section{Unmet needs in CMR}

This session aimed to bridge the gap between current practice of CMR and the level where this technique should ideally be. A top ten list identified the most important needs in CMR to be addressed by future studies, including, for example, CMRS or molecular imaging. The complexity of CMR in clinical practice and the required user expertise were identified as major shortcomings of this versatile technique, currently preventing a more widespread use and disadvantaging CMR against CT. The industry and several professional groups are currently addressing this issue.

\section{Interventional CMR}

At the interventional cardiovascular MRI workshops, numerous innovations and novel approaches were presented, such as interactive, real-time MRI workstations for multi-purpose use for interventional studies (Dr. Juan Santos, Stanford University, Stanford, CA, USA and Dr. Michael Guttman, NIH, Bethesda, MD, USA), dualmodality fusion systems (Dr. Perry Radau, Sunnybrook Hospital, Toronto, Canada), new catheter, control and tracking systems (Dr. Albert Lardo, Johns Hopkins University, Baltimore, MD, USA and Dr. Eigil Samset, Brigham and Women's Hospital, Boston, MD, USA and University of Oslo, Oslo, Norway). Dr. Amish Raval of University of Wisconsin at Madison emphasized that realtime interventional CMR will be useful not to complicate successful standard procedures but to enable or enhance otherwise complex procedures. Dr. Dana Peters of Beth Israel Deaconess Medical Center, Boston, MD showed that the volume of the ablation-related scar as assessed by CMR predicted success or failure of radiofrequency ablation of atrial fibrillation.

During oral abstract presentations, Dr. Aaron Bell of Kings College London described MRI cardiac catheterization in patients with single ventricle physiology congenital heart disease (209 [A70]). Unlike available modalities, CMR catheterization measured differential pulmonary vascular resistances when there were multiple sources of pulmonary flow, invaluable in surgical management. Dr. Elmar Spuentrup of University of Cologne, Germany reported on preclinical experiments with EP-3600 collagen-specific gadolinium-based contrast for myocardial stress imaging (207 [A68]). In a pig myocardial stenosis model during adenosine stress, EP-3600 exhibited differential myocardial accumulation and retention for over 20 minutes. If further corroborated, this might enable steady-state highresolution perfusion imaging, or even possibly treadmill exercise stress MRI. Dr. Kanishka Ratnayaka of National Institutes of Health, Bethesda, $\mathrm{MD}$ and Childrens National Medical Center described fusion of CMR imaging and live X-ray to guide antegrade catheter treatment of spontaneous membranous ventricular septal defect in swine (109 [A10]). X-ray simplified the procedure and halved radiation compared with conventional techniques.

Dr. Henry Halperin of Johns Hopkins University, Baltimore, MD presented early clinical electrophysiology catheter experiments conducted wholly under CMR in human subjects. He also demonstrated visually stunning realtime images combined with 3-dimensional volumetric renderings from prior examinations. 


\section{Congenital CMR \\ CMR of tetralogy of fallot}

A focus of CMR in congenital heart disease was a session on CMR in Tetralogy of Fallot. CMR was shown as an important technique for monitoring the major residual lesion after total correction - pulmonary regurgitation. Monitoring of right ventricular volumes and ejection fraction is critical in determining the need and timing of pulmonary valve replacement (PVR). Dr. Karen Ordovas (Department of Radiology, University of California School of Medicine, San Francisco, CA, USA) indicated that when the end systolic volume index exceeded $95 \mathrm{ml} /$ $\mathrm{m}^{2}$, the total and effective ejection fractions were reduced below $40 \%$ and $25 \%$ respectively. Dr. Alexander van Straten from Leiden University Medical Center, Leiden, NL, showed in PVR that RVEDV and RVESV decreased in most patients at 8 months postoperatively, with little further reduction at 20 months. A favorable postoperative response was predicted by a preoperative RVEDV index of less than $160 \mathrm{ml} / \mathrm{m}^{2}$. Recurrent pulmonary regurgitation occurred in about $44 \%$ of patients. Dr. Andrew Taylor of Great Ormond Street Hospital in London, UK reported that transcatheter PVR was only suitable if the pulmonary annulus was $22 \mathrm{~mm}$ or less. CMR was found to be important in determining the size and shape of the RV outlet region in order to determine the suitability of transcatheter PVR. CMR was especially useful for determining if the left coronary artery might be jeopardized during the procedure.

\section{What's new in congenital CMR?}

In this session on novel approaches in congenital CMR, Dr. Heynric Grotenhuis of Leiden University Medical Center, Leiden reported on the use of phase contrast CMR to demonstrate reduced aortic distensibility and aortic elasticity in patients after arterial switch procedure. Aortic root dilation and reduced elasticity were associated with aortic regurgitation and reduced left ventricular function (215 [A76]). Dr. Craig Broberg of Oregon Health and Science University, Portland proposed a previously described Look-Locker technique to estimate the distribution volume of gadolinium chelate in the myocardium. An increased distribution volume in Eisenmenger patients and patients years after repair of transposition was interpreted as evidence of diffuse myocardial fibrosis (216 [A77]). This diffuse fibrosis is less readily recognized than replacement fibrosis occurring after myocardial infarction.

\section{Best Technologist Abstract}

Ricardo Wage (Royal Brompton Hospital, London, UK) received this year's best technologist abstract award for his presentation on technical considerations for the CMR assessment of the mitral valve.

\section{Best Manuscript Award of the Journal of Cardiovascular MR (JCMR)}

Dr. Ann Bolger (UCSF, San Francisco, CA and Linköping University, Sweden) received the Gerald Pohost Best Manuscript Award of the Journal of Cardiovascular Magnetic Resonance $(J C M R)$ for her paper "Transit of Blood Flow through the Human Left Ventricle Mapped by Cardiovascular Magnetic Resonance" [2]. Editor-in-Chief Dr. Dudley Pennell used the opportunity to remind readers that the $J C M R$ is now available as an open access journal (online at http://jcmr-online.com).

\section{Conclusion}

Among this year's highlights of the Annual Sessions of the SCMR were scientific reports on CMR-based risk assessment, non-contrast tissue characterization, $3 \mathrm{~T}$ data, and interventional CMR.

In 2009, the $12^{\text {th }}$ Annual Sessions will be held from February 1 to February 3 in Orlando, Florida.

Additional details on previous and future meetings can be found on the SCMR website http://www.scmr.org.

\section{Financial support}

No financial support was received for this manuscript.

\section{List of abbreviations used}

CMR: Cardiovascular Magnetic Resonance; CMRS: Cardiovascular Magnetic Resonance Spectroscopy; EDV: EndDiastolic Volume; ESV: End-Systolic Volume; LGE: Late Gadolinium Enhancement; LV: Left Ventricle; MDCT: Multi-Detector Computed Tomography; MRA: Magnetic Resonance Angiography; PVR: Pulmonary Valve Replacement; RV: Right Ventricle; RF: Radiofrequency; SAR: Specific Absorption Ratio; SPECT: Single-Photon Emission Computed Tomography; SSFP: Steady-state free precession; T: Tesla.

\section{Acknowledgements}

The authors are most grateful for the contributions of Drs. Robert Lederman, Raymond Kwong, and Jeanette Schulz-Menger.

Abstracts in the text are referenced by the original number by which they were presented at the conference (viewable in the SCMR 2008 abstract document on the SCMR website http://www.scmr.org) accompanied by a number prefixed A that identifies the published abstract in the Journal of Cardiovascular Magnetic Resonance at http://jcmr-online.com/supplements/I0/ SI.

\section{References}

I. Schwitter J, Wacker CM, van Rossum AC, Lombardi M, Al-Saadi N, Ahlstrom H, Dill T, Larsson HB, Flamm SD, Marquardt M, Johansson L: MR-IMPACT: comparison of perfusion-cardiac magnetic resonance with single-photon emission computed tomography for the detection of coronary artery disease in a multicentre, multivendor, randomized trial. Eur Heart J 2008, 29:480-489. 
2. Bolger AF, Heiberg E, Karlsson M, Wigström L, Engvall J, Sigfridsson A, Ebbers T, Kvitting JP, Carlhäll CJ, Wranne B: Transit of blood flow through the human left ventricle mapped by cardiovascular magnetic resonance. J Cardiovasc Magn Reson 2007, 9:74I-747.

Publish with Biomed Central and every scientist can read your work free of charge

"BioMed Central will be the most significant development for disseminating the results of biomedical research in our lifetime. " Sir Paul Nurse, Cancer Research UK

Your research papers will be:

- available free of charge to the entire biomedical community

- peer reviewed and published immediately upon acceptance

- cited in PubMed and archived on PubMed Central

- yours - you keep the copyright

Submit your manuscript here:

http://www.biomedcentral.com/info/publishing_adv.asp 